\title{
Tumor necrosis factor alpha inhibitors and demyelinating disease: what is behind it?
}

\author{
Eugeniusz Józef Kucharz ID , Anna Kotulska-Kucharz ID \\ Department of Internal Medicine, Rheumatology and Clinical Immunology, Medical University of Silesia, Katowice, Poland
}

\section{Introduction}

The development of tumor necrosis factor alpha (TNF- $\alpha$ ) inhibitors is probably one of the most significant advancement in the management of a number of inflammatory rheumatic disorders to date. High efficacy of the treatment is associated with relatively rare and very heterogeneous adverse reactions. The reactions are gradually recognized with an increase in number of the patients receiving medication and prolonged period of the drug usage. Neurological adverse events in patients receiving TNF- $\alpha$ inhibitors are not common. This finding emergence a question of coincidental occurrence of the nervous system involvements as opposite to suggestion of induction of the nervous system disease or unmasking of latent disease due to the drug administration.

Potential association of anti-TNF- $\alpha$ therapy with demyelination is one of neurological adverse reaction, and is an important concern for every day clinical practice of rheumatologist as well as is an interesting problem from pathophysiological point of view.

\section{Incidence of TNF- $\alpha$ inhibitor-associated demyelinating disease}

Demyelinating events have been reported in association with the use of TNF- $\alpha$ inhibitors mostly as case reports or small series of cases. Food and Drug Administration issued a warning label on all anti-TNF- $\alpha$ drugs after a phase II study on lanercept, TNF- $\alpha$ inhibitor that had been suggested to be beneficial in patients with multiple sclerosis. The drug was found to facilitate the disease exacerbation and the trial was discontinued. Subsequently, all the drugs of the class have been labeled with the same warning.

Incidence of demyelinating disease in patients treated with TNF- $\alpha$ inhibitors remains unknown but seem to be very low. Population-based cohort study from Denmark and Sweden revealed lack of association between TNF- $\alpha$ inhibitor treatment of rheumatoid arthritis and risk of a neuroinflammatory event for the Swedish cohort, and an elevated but not significantly increased risk in the Danish cohort. In patients with ankylosing spondylitis or psoriatic arthritis a 50 per cent increased risk of a neuroinflammatory event among patients exposed to TNF- $\alpha$ inhibitors compared with those not exposed was shown [1].

There is no clear explanation of the difference in the risk of the neurological involvement between patients with rheumatoid arthritis and inflammatory spondyloarthropathy. Different genetic profiles and different distribution of age and gender between the groups of the patients was suggested as a possible explanation of the difference. A non-significant increased risk of demyelinating events following exposure to TNF- $\alpha$ inhibitors was reported in the US patients with rheumatoid arthritis in 2010 [2]. Later, Etminan et al. [3] described a more than twofold increase in risk of peripheral neuropathy in cumulated group of rheumatic patients with inflammatory arthritides. In general, demyelination is estimated to occur between 0.03 and 0.2 per cent of patients receiving TNF- $\alpha$ inhibitors [4].

\section{Pathogenesis of demyelination}

The mechanism of demyelination associated with TNF- $\alpha$ inhibitors remains largely unknown. A few hypothetical phenomena have been suggested [5]. TNF- $\alpha$ in rheumatology is considered as the main proinflammatory cytokine responsible for sequence of inflammation within the joints. Down regulation of TNF- $\alpha$ was shown to limit pathologic inflammation also in the skin, and improve psoriatic plaque [6]. In fact, TNF- $\alpha$ plays much move complex function and is involved in several regulatory pathways.

TNF- $\alpha$ was found to modulate the autoreactive T cells reactivity to self-antigens. Thus, reduction of TNF- $\alpha$ level is associated with enhanced activity of autoreactive 
T-cells. This phenomenon may be responsible for development of drug-induced lupus-like disease in some patients treated with TNF- $\alpha$ inhibitors. It is believed that the same mechanism plays a role in autoimmune damage of the myelin sheaths. Additionally, interferon- $\gamma$ release accompanying T-cell activation enhanced demyelination.

Administration of TNF- $\alpha$ inhibitors can result in a decrease in immune response. Discontinuation of the medication can be associated with immune reconstitution syndrome and manifested as autoimmune demyelination. Another mechanism of the observed phenomena links demyelination with deposition of immune complexes consisting of TNF- $\alpha$ and TNF- $\alpha$ inhibitors. Deposition of the complexes can cause vasculitis, and indirectly stimulate ischemic demyelination.

The list of hypothetical mechanisms of TNF- $\alpha$ inhibitor associated demyelination also includes influence upon a transmembrane molecule of TNF- $\alpha$ and impairment of the proliferation of immature oligodendrocytes and remyelination.

Genetic variants of TNF- $\alpha$ receptor superfamily $1 \mathrm{~A}$ which are associated with multiple sclerosis were not found to be associated with TNF- $\alpha$ inhibitors-induced demyelinating complications [7].

\section{Clinical presentation}

Demyelination within the nervous system is in most of the cases asymptomatic and discovered incidentally. In some patients it can be a cause of symptoms from mild to significantly severe ones. Seror et al. [8] in the French national survey revealed that the peripheral nervous system involvements are twice common as compare with the central system manifestations. Cortese et al. [9] classified demyelinating events associated with TNF- $\alpha$ inhibitor administration in the following groups: patients with isolated episode of demyelination, new onset of multiple sclerosis, and worsening of pre-existing multiple sclerosis. The last group is resulted from administration of the drug against recommendations which indicate for avoidance of such medication in patients with suspicion or diagnosis of multiple sclerosis.

The central nervous system involvements include optic neuritis, cranial nerve paresis and a large scope of multiple sclerosis-related symptomatology. The course of overt demyelinating disease is heterogeneous. After initial progression, clinical picture can be stable, and discontinuation of the medication in majority of patients resulted in regression of neurological symptoms. On the other hand, in some patients the symptoms indicate for onset or unmasking of multiple sclerosis or other disorder characterized by demyelination [10].
Administration of TNF- $\alpha$ inhibitors in rare cases resulted in overt multiple sclerosis. It is uncertain, if the cases represent de novo inflammatory disease developed in predisposed individuals or exacerbation of preexisting latent disorder. The course of multiple sclerosis associated with TNF- $\alpha$ inhibitors seems to be similar to idiopathic cases, and can be managed with typical medication.

In most of the cases of TNF- $\alpha$ inhibitor-induced demyelination discontinuation of the causative medication is sufficient therapy but administration of corticosteroids and/or intravenous immunoglobulins are required in a part of the patients. Rechallenge with TNF- $\alpha$ inhibitors results almost always a flare and is contraindicated. Secukinumab may be considered as a valid treatment option [8].

\section{Conclusions}

Neurological demyelinating complications are rare in patients treated with TNF- $\alpha$ inhibitors but rheumatologists should be aware of them. Patients with previous demyelinating events should not be treated with TNF- $\alpha$ inhibitors or if benefits of such medication overweigh the risk of progress in demyelination, the management of the patient should be done under special supervision and early detection of neurological complications. Other biological drugs than TNF- $\alpha$ inhibitors can be considered as safe alternative if possible.

The authors declare no conflict of interest.

\section{References}

1. Kopp TI, Delcoigne B, Arkema EV, et al. Risk of neuroinflammatory events in arthritis patients treated with tumor necrosis factor alpha inhibitors: collaborative population-based cohort study form Denmark and Sweden. Ann Rheum Dis 2020; 79: 566-572, DOI: 10.1136/annrheumdis-2019-216693.

2. Bernatsky S, Renoux C, Suissa S. Demyelinating events in rheumatoid arthritis after drug exposures. Ann Rheum Dis 2010; 69: 1691-1693, DOI: 10.1136/ard.2009.111500.

3. Etminan M, Sodhi M, Samii A, et al. Tumor necrosis factor inhibitors and risk of peripheral neuropathy in patients with rheumatic diseases. Semin Arthritis Rheum 2019; 49: 10831086, DOI: 10.1016/j.semarthrit.2018.09.006.

4. Bosch X, Saiz A, Ramos-Casals M, BIOGEAS Study Group. Monoclonal antibody therapy-associated neurological disorders. Nature Rev Neurol 2011; 7: 165-172, DOI: 10.1038/nrneurol.2011.1.

5. Kaltsonoudis E, Voulgari PV, Konitsiotis S, Drosos AA. Demyelination and other neurological adverse events after anti-TNF therapy. Autoimm Rev 2014; 13: 54-58, DOI: 10.1016/j.autrev. 2013.09.002.

6. Zhu TH, Nakamura M, Abrouk M, et al. Demyelinating disorders secondary to TNF-inhibitor therapy for psoriasis: a review. 
J Dermatol Treat 2016; 27: 406-413, DOI: 10.3109/ 09546634 2015.1136385.

7. Bitoun S, Miceli-Richard C, Verstuyft C, et al. Frequency of tumor necrosis factor alpha receptor superfamily $1 \mathrm{~A}$ multiple sclerosis-associated variants in patients with rheumatoid arthritis with anti-tumor necrosis factor therapy-related demyelinating complications. Ann Rheum Dis 2018; 77: 1835-1836, DOI: 10.1136/annrheumdis-2018-213183.

8. Seror R, Richez C, Sordet C, et al. Pattern of demyelination occurring during anti-TNF- $\alpha$ therapy: a French national sur- vey. Rheumatology (Oxford) 2013; 52: 868-874, DOI: 10.1093/ rheumatology/kes375.

9. Cortese R, Prosperini L, Stasolla A, et al. Clinical course of central nervous system demyelinating neurological events associated with anti-TNF-therapy. J Neurol 2021, DOI: 10.1007/ s00415-021-10460-6 [Online ahead of print].

10. Kotyla P, Śliwińska-Kotyla B, Kucharz EJ. Treatment with infliximab may contribute to the development of peripheral neuropathy among the patients with rheumatoid arthritis. Clin Rheumatol 2007; 26: 1595-1596, DOI: 10.1007/s10067-007-0657-3. 